\title{
CHANNEL BORROWING PROJECT BASED ON SURPLUS VALUE
}

\author{
Hui Zeng * Yuanwei JING * \\ Georgi M. Dimirovski ${ }^{* *, * * *}$
}

\author{
* Northeastern University, Faculty of Information Science \\ and Engineering, Shenyang, Liaoning 110004 \\ P.R. of China \\ E-mail: zh6135@163.com; ywjjing@163.com \\ ** Dogus University of Istanbul, Faculty of Engineering, \\ Computer Engg. Dept. Acibadem, Zeamet Sk. 21, Kadikoy, \\ TR-34722 Istanbul, Rep. of Turkey E-mail: \\ gdimirovski@dogus.edu.tr; Fax: +90-216-327-9631; Tel: \\ +90-32\%-1104*Authors and address for correspondence \\ *** SS Cyril and Methodius University, Faculty of Electrical \\ Engineering Skopje, MK-1000, Rep. of Macedonia
}

\begin{abstract}
The problem of channel choice is investigated from the point of view of the users' benefit function and a novel solution is proposed. When users possess a channel, the minimum-lending-priority channel is assigned from nominal channel in one's own cell firstly. If there is no vacancy, it is borrowed from the neighborhood cells. When users release channel, the minimum cost function channel is chosen. Nash equilibrium and Stackelberg strategy of the game theory are employed to lead the users to choose the maximal surplus value scheme. This decision and control scheme can not only enhance channel utilization and reduce the blocking rate, but also guarantee the satisfied surplus value. Simulation results are given to illustrate the proposed method. Copyright (c) 2005 IFAC
\end{abstract}

Keywords: Communication systems, channel borrowing, quality of service, surplus value, game theory, incentives.

\section{INTRODUCTION}

With the rapid development of Global System of Mobile (GSM) Communications, the number of network users increases steadily and portfolio expends continuously. And along with this, the relative lack of system resources inevitably affects the network communications. It is a bottleneck of development in mobile communications to increase spectrum utilization of resource in existence. Channel assignment is an effective way to solve it (Yeung and Yum, 1994; Li, et al., 2000), and it has been investigated broadly up to the present days. There is a considerable practical importance to study rather deeply the channel assignment problem.

There are a number of channel assignment methods (Katzela and Naghshineh, 1996). Fixed Channel Assignment (FCA) can not deal with hotspot problems. Dynamic Channel Assignment (DCA) and Hybrid Channel Assignment (HCA) solve it to some degree, but the minimum duplicate distance of system can not be guaranteed. Channel assignment is an optimization decision and control problem essentially, and the key point is to get op- 
timal solution. So, it is emergent to establish channel assignment model with good performance and high-speed arithmetic. Channel borrowing allows channel to be borrowed from neighborhood while channel locking is not needed. This channel borrowing project is easily carried out and has high spectrum utilization. Non-symmetrical increase of GSM portfolio makes channel assignment strategy be studied deeply as an important way to deal with non-symmetrical operation. At present, there are mostly simple borrowing, priority borrowing and locking borrowing with direction, and so on (Jiang, et al., 1994).

Channel borrowing is a kind of methods to enhance performance of Fixed Channel Assignment. The channel borrowing project is based on the network frequency rule. Every cell has nominal channel, and similar capacity performance of Dynamic Channel Assignment can be achieved when portfolio overloads. The cell is classified as "cold" state and "hot" state, which improves QoS of the system and does not reduce agility degree. Combination of local and integer information reduces service deviation (Dang, et al., 2002). Channel borrowing supposes that the cell can not load operation of its own while neighbor keeps very low degree. Then, the channel of disturbance restriction satisfied is borrowed to improve system resource utilization.

Cheng, et al., (2001) discussed channel assignment based on priority borrowing. The project can reduce blocking rate and improve capacity in hotspot evidently, and guarantee well applicability to GSM system. Only priority is considered, which is not effective in assort with users choice of channel, while benefit function as a guarantee can be more easily in leading users and more closer to fact. Zhang and Yum, (1989), and Baiocchi, et al., (1985), have studied channel assignment based on cost function, by means of which the assignment strategy is realized. However, local information usage only can not properly choose from the whole system, which is an apparent deficiency of these methods.

The paper studies "Channel borrowing project" based on surplus value. Nominal channels are assigned in some scale according to statistic and forecast data (Guo, et al., 2002). The rest of this paper is written as follows. In Section 2 the definition of the appropriate model is given.

\section{MODEL DESCRIPTION}

A communication network with a set of $J$ resources, where $u_{i}\left(x_{i j}\right)$ is the utility function. User $i$ uses the network with rates $x_{i j}$ in $j$ priority, $j=$ $1,2 \cdots M$, including the possessing at first and releasing channel later.Quantity $l_{j}$ represents possessing price and $\lambda_{j}$ is releasing price in different priority, where $j$ is priority. As in Jing, et al., (2001), surplus function of system can be described as follows:

$$
c_{i}\left(x_{i j}\right)=u_{i}\left(x_{i j}\right)-\sum_{j=1}^{M} l_{j} x_{i j}-\sum_{j=1}^{M} \lambda_{j} x_{i j}
$$

When call is coming, the minimum-lending-priority nominal channel in the cell is assigned to the call. If there is not vacant, it is borrowed from the neighborhood. Quantity $l_{j} x_{i j}$ expresses the payment price of possessing the channel. The cost is minimal when it possesses channels in one's own nominal cell, otherwise punishment price or borrowing price must be paid. It is the same on release condition, just cost value is not different. Therefore, channel of the minimal cost value is chosen to guarantee benefit of users. But, it can not always assort with each other well to the system of multi users. Stackelberg strategy and Nash equilibrium conception in game theory are adopted to lead users using channels in some rates, which can realize requirement of users and guarantee the benefit.

Next, some useful concepts of the game theory are introduced in here. The network should be the leader in the strategy, and the users should represent the followers who act at the Nash equilibrium. Hence, if the leader wants users to be at the rates which are arranged by the network, the leader must have the leadership in the game. This is indicated in the following Stackelberg strategy.

$$
\xi_{i}\left(x_{i j}\right)=l_{j}+\lambda_{j}+p_{i}\left(x_{i j}\right)-p_{i}\left(x_{i j}^{a}\right)
$$

where, $x_{i j}^{a}$ is the desired channel rate of network, and $a$ expresses the desired point of network, $p_{i}\left(x_{i j}^{a}\right)$ is the payment price of using the channel, $p_{i}\left(x_{i j}\right)$ is a function to be determined. Users must pay punishment price for departure from desired point of network. However, the strategy can produce small deviation to guarantee satisfied surplus.

\section{THE CASE OF ELASTIC TRAFFIC}

\subsection{Linear incentive strategy}

In this section, the linear function is considered as the Stackelberg incentive strategy to force users to act at the point $x_{i j}^{a}$, supposing $p_{i}\left(x_{i j}\right)=q_{i} x_{i j}$ and replacing $l_{j}+\lambda_{j}$ by $\xi_{i}\left(x_{i j}\right)$, then 


$$
\begin{aligned}
c_{i}\left(x_{i j}\right)= & u_{i}\left(x_{i j}\right)-\sum_{j=1}^{M} x_{i j}\left(l_{j}+\lambda_{j}\right) \\
& -\sum_{j=1}^{M} q_{i}\left(x_{i j}-x_{i j}^{a}\right) x_{i j}
\end{aligned}
$$

is surplus function of users, where $q_{i}$ is an unknown quantity. Calculating the derivative of (3) with respect to $x_{i j}$, setting it be zero, and taking the value of $x_{i j}$ at $x_{i j}^{a}$, then

$$
q_{i}=\frac{\dot{u}_{i}\left(x_{i j}^{a}\right)-\sum_{j=1}^{M} l_{j}-\sum_{j=1}^{M} \lambda_{j}}{x_{i j}^{a}}
$$

And so, the strategy should be

$$
\begin{aligned}
\xi_{i}\left(x_{i j}\right)= & l_{j}+\lambda_{j}+ \\
& \frac{\dot{u}_{i}\left(x_{i j}^{a}\right)-\sum_{j=1}^{M}\left(l_{j}+\lambda_{j}\right)}{x_{i j}^{a}}\left(x_{i j}-x_{i j}^{a}\right)
\end{aligned}
$$

Leading $\xi_{i}\left(x_{i j}^{a}\right)$ back to the former model

$$
\xi_{i}\left(x_{i j}^{a}\right)=l_{j}+\lambda_{j}
$$

and

$$
\begin{aligned}
\operatorname{argmax}\left[u_{i}\left(x_{i j}\right)\right. & -\sum_{j=1}^{M}\left(x_{i j}\left(l_{j}+\lambda_{j}\right)\right. \\
& \left.\left.-q_{i}\left(x_{i j}-x_{i j}^{a}\right) x_{i j}\right)\right]=x_{i j}^{a}
\end{aligned}
$$

Note that $p_{i}\left(x_{i}\right)=q_{i} x_{i j}$ can be linear incentive strategy just as shown in model 5 and 6 , then

$$
\begin{aligned}
& u_{i}\left(x_{i j}^{a}\right)-\sum_{j=1}^{M} x_{i j}^{a}\left(l_{j}+\lambda_{j}\right) \geq u_{i}\left(x_{i j}\right)- \\
& \sum_{j=1}^{M}\left[x_{i j}\left(l_{j}+\lambda_{j}\right)+q_{i}\left(x_{i j}-x_{i j}^{a}\right) x_{i j}\right]
\end{aligned}
$$

Function (3) is a second-order function of $x_{i j}$, and the coefficient of the second order item is negative, then the function picture placket is down. $x_{i j}^{a}$ is the only extreme point, so, the maximum value is got at $x_{i j}^{a}$. On the other hand, because of $\left(x_{i j}-\right.$ $\left.x_{i j}^{a}\right) x_{i j}>0$, it follows $q_{i}\left(x_{i j}-x_{i j}^{a}\right) x_{i j}>0$, and the above conclusion can be obtained naturally. That is, the maximum value of users can be achieved in using channel at network desired rates.

In ordinary network communications, it does not sacrifice communication quality to obtain max surplus value. The strategy guarantees users benefit, and transfers information in some rule, which not only avoids congestion but also uses bandwidth resource adequately. Users choose channel around its own cell, transfer requirement is satisfied and small service deviation is guaranteed.

Cheng, et al., (2001) have discussed channel assignment based on priority borrowing, each nominal channel of a cell has its borrowing priority. The paper also considers priority problem of nominal channel, big or small of priority is expressed by price so that judgment and choice of priority are avoided. The other hand, it is difficult to distinguish fixed channel and dynamic channel in software realization, while the difference is expressed by price can make complex process simplified. Price scale lets users know the surplus condition directly and makes network manage users easily, however it has to make use of game theory effectively.

\subsection{Non-linear incentive strategy}

In the section, a non-linear incentive strategy is considered

$$
p_{i}\left(x_{i j}\right)=\begin{array}{cl}
\frac{\left(\lambda_{j}+l_{j}\right)\left(x_{i j}^{a}-x_{i j}\right)}{x_{i j}}, & x_{i j}<x_{i j}^{a} \\
0, & x_{i j}=x_{i j}^{a}(8) \\
\frac{u_{i}\left(x_{i j}\right)-u_{i}\left(x_{i j}^{a}\right)}{x_{i j}}, & x_{i j}>x_{i j}^{a}
\end{array}
$$

Obviously, if $x_{i j}=x_{i j}^{a}$, strategy (2) becomes $\xi_{i}\left(x_{i j}\right)=\lambda_{j}+l_{j}$ and satisfies model (5). Then (8) is substituted into (1) with the structure of $\xi_{i}\left(x_{i j}\right)=\lambda_{j}+l_{j}+p_{i}\left(x_{i j}\right)-p_{i}\left(x_{i j}^{a}\right)$. Then it follows at once:

(a)If $x_{i j}<x_{i j}^{a}, p_{i}\left(x_{i j}\right)=\frac{\left(\lambda_{j}+l_{j}\right)\left(x_{i j}^{a}-x_{i j}\right)}{x_{i j}}$,

then $c_{i}\left(x_{i j}\right)=u_{i}\left(x_{i j}\right)-\sum_{j=1}^{M}\left(l_{j} x_{i j}^{a}+\lambda_{j} x_{i j}^{a}\right)$,

so, $c_{i}\left(x_{i j}\right)<c_{i}\left(x_{i j}^{a}\right)$

(b)If $x_{i j}>x_{i j}^{a}, p_{i}\left(x_{i j}\right)=\frac{u_{i}\left(x_{i j}\right)-u_{i}\left(x_{i j}^{a}\right)}{x_{i j}}$, then

$c_{i}\left(x_{i j}\right)=u_{i}\left(x_{i j}^{a}\right)-\sum_{j=1}^{M}\left(l_{j} x_{i j}+\lambda_{j} x_{i j}\right)$,

so, $c_{i}\left(x_{i j}\right)<c_{i}\left(x_{i j}^{a}\right)$

In both cases, it is shown that $c_{i}\left(x_{i j}\right)<c_{i}\left(x_{i j}^{a}\right)$ , which indicates the satisfaction of the second condition for incentive strategy. The non-linear incentive strategy considers $x_{i j}^{a}$ as dividing point to discuss surplus function. From the above analysis, it can be seen that maximum surplus value still is attained at $x_{i j}=x_{i j}^{a}$.

Linear incentive and non-linear incentive strategy have been found to prove that the maximum surplus value is achieved at $x_{i j}=x_{i j}^{a}$.Pricing control is a way to guarantee unification of multi-user 
system, even departure from the exact solution of one user can affect other users. In this paper, Stackelberg Strategy and Nash equilibrium conceptualization are adopted so that the users are lead to choose channels in the cell or the neighbor cell, and possess some rates of releasing channels, which satisfies the requirement of users and guarantees the benefit.

\section{THE CASE OF NON-ELASTIC TRAFFIC}

In a practical communication network, however, the delay occurs very often. The more the traffic rate is close to the capacity of resources, the higher the delay will be. Therefore, the utility function can not be always increasing. In this section, the problem is to be dealt with that the utility function is protruding but no longer increasing.

Let it be assumed $x_{i j}^{*}$ is the optimal rates of network, namely $\dot{u}_{i}\left(x_{i j}^{*}\right)=0$, and also that $x_{i j}^{u}$ is the optimal rate for the user, namely $\dot{u}_{i}\left(x_{i j}^{u}\right)-l_{j}-$ $\lambda_{j}=0$. Obviously, if $l_{j}+\lambda_{j} \neq 0$ then $x_{i j}^{u} \neq x_{i j}^{*}$. Users benefit function and well-balanced system must be guaranteed for non-elastic traffic problems, and so the incentive strategy is to be found to force users to act at the point $x_{i j}^{*}$.

If the linear function here again is used, then user surplus function is:

$$
\begin{aligned}
c_{i}\left(x_{i j}\right) & =u_{i}\left(x_{i j}\right)- \\
& \sum_{j=1}^{M} x_{i j}\left[\left(l_{j}+\lambda_{j}\right)+q_{i}\left(x_{i j}-x_{i j}^{*}\right)\right]
\end{aligned}
$$

where $q_{i}$ is an unknown quantity. Calculating the derivative of (9) with respect to $x_{i j}$, letting it be zero, and taking the value of $x_{i j}$ at $x_{i j}^{*}$, then $q_{i}=-\frac{\left(l_{j}+\lambda_{j}\right)}{x_{i j}^{*}}$. It can be easily shown that there is an incentive Stackelberg strategy under the condition $l_{j}+\lambda_{j}<-\frac{\ddot{u}_{i}\left(x_{i j}^{*}\right) x_{i j}^{*}}{2}$, which means that the regular price should be determined in a reasonable range.

Portfolio is increasing and network capacity is limited, so justice and efficiency should be guaranteed in network communications. It is the same to nonelastic traffic. Channel borrowing scheme in the paper provides each user with equal opportunity to use network bandwidth, and regulate transfer information rates according to usage condition of network, so justice and efficiency both are considered.

\section{SIMULATION RESULTS}

\subsection{Elastic traffic case}

Consult the example in reference Zhang and Yum, (1989) and take $u_{i}\left(x_{i j}\right)=m_{i} \log x_{i j}$ as well as set definite capacity of network be $\mathrm{C}=10$. Figure 1 depicts the results of user with $m_{i}=5, \lambda_{j}=0.5$ and $l_{j}=0.1$ for different points $x_{i j}^{a}=6$ and $x_{i j}^{a}=8$ respectively. The maximum is really at $x_{i j}^{a}$ in each case.

Next, the following expression is considered, which defines the non-linear incentive strategy

$$
\xi_{i}\left(x_{i j}\right)=\begin{array}{cl}
0.6+\frac{0.6\left(6-x_{i j}\right)}{x_{i j}}, & \\
0.6, & x_{i j}<6 \\
0.6+\frac{5\left(\log x_{i j}-\log 6\right)}{x_{i j}}, & x_{i j}=6
\end{array}
$$

The maximum of users is obtained at the point $x_{i j}=6$ just as it is depicted in Figure 2 .

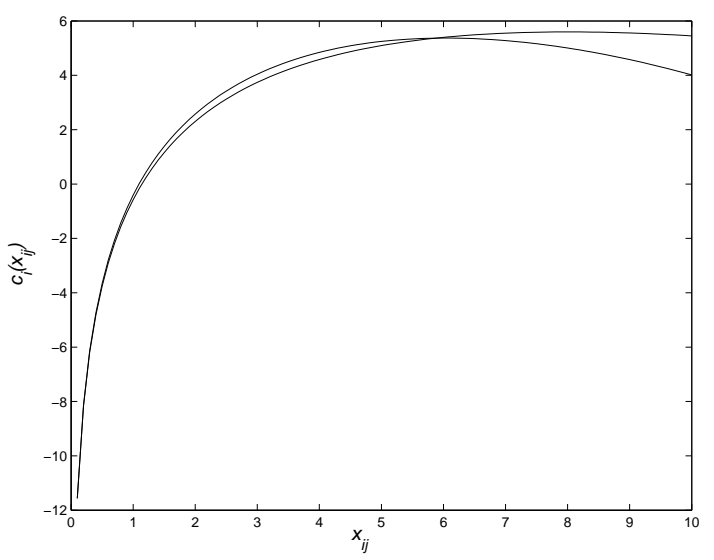

Fig. 1. curve of the functions for network user

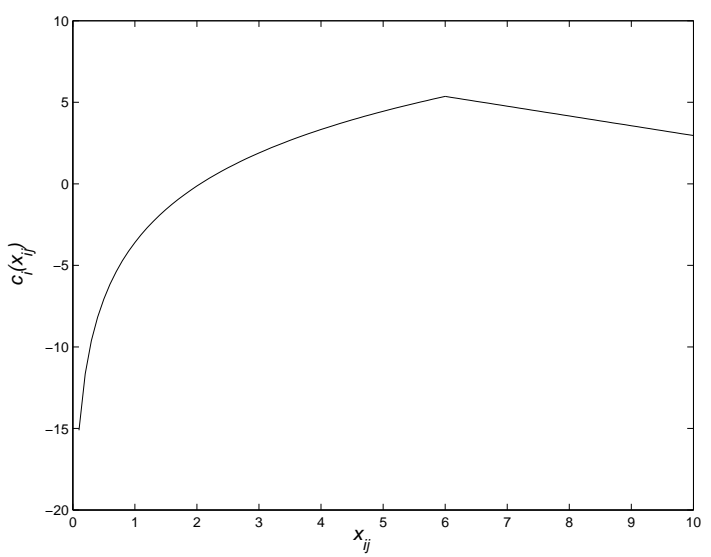

Fig. 2. curve of user benefit function in subsection strategy

Figure1 and figure 2 show that the maximum surplus value can be achieved at $x_{i j}^{a}$, which accords 
with the demonstrated result $c_{i}\left(x_{i j}\right)<c_{i}\left(x_{i j}^{a}\right)$, so the effectiveness of game theory in leading users is proved. On the other hand, the guaranteed surplus value makes users choose and borrow channel reasonably, so the whole network system runs more stably.

\subsection{Non-elastic traffic case}

Now, let it brought to focus the case of $u_{i}\left(x_{i j}\right)=$ $m_{1} \log x_{i j}+m_{2} \log \left(c-x_{i j}\right)$ for the non-elastic traffic. Hence, when $x_{i j}^{*}=2$, it is $\left(m_{1}, m_{2}\right)=$ $(2,8)$, when $x_{i j}^{*}=5,\left(m_{1}, m_{2}\right)=(5,5)$, and when $x_{i j}^{*}=8,\left(m_{1}, m_{2}\right)=(8,2)$.

Then $x_{i j}^{u}=\operatorname{argmax}\left[u_{i}\left(x_{i j}\right)-\sum_{j=1}^{M} x_{i j}\left(l_{j}+\lambda_{j}\right)\right]$ is not consistent with $x_{i j}^{*}$ for the existence of punishment price, hence the linear incentive strategy is adopted. For $x_{i j}^{*}=5$, the corresponding parameter is $q_{i}=-0.12$; then incentive function is $p_{i}\left(x_{i j}\right)=-0.12 x_{i j}$, and satisfies $0.6=\lambda_{j}+$ $l_{j}<-\frac{\ddot{u}_{i}\left(x_{i j}^{*}\right) x_{i j}^{*}}{2}=1$. Therefore $u_{i}\left(x_{i j}^{u}\right)=10.68$ is obtained when $u_{i}\left(x_{i j}\right)=5 \log x_{i j}+5 \log (10-$ $\left.x_{i j}\right)$. However, $u_{i}\left(x_{i j}^{*}\right)=13$ when the corresponding strategy is adopted. Therefore, the users will choose $x_{i j}^{*}=5$ for non-elastic traffic, which guarantees a stable system and the users benefit.

\section{CONCLUSIONS}

Channel borrowing problem based on surplus value has been solved in this paper. When call is coming, the minimum-lending-priority nominal channels in the cell are assigned to the call. If there is not vacant, it is borrowed from the neighborhood. When users release the channel, the minimum cost function channel is chosen. Nash equilibrium and Stackelberg strategy in game theory are used to lead users to choose the maximal surplus value scheme. The scheme can not only enhance channel utilization, reduce blocking rate and service deviation, but also guarantee the satisfied surplus value.

The FCA reduces agility performance which can not satisfy non-symmetrical portfolio, DCA and HCA can deal with hotspot problems, but software realization is difficult and calculating process is complex. The rates of FCA and DCA are assigned approximately according to statistic and forecast data of portfolio, which reduces calculating process greatly, and saves time and responds quickly. It is seen from the model that the channel borrowing project based on surplus value makes the channel choice on priority and sets different price for different priority. Time delay and nontime delay operation can choose different priority to transfer information, requirement is satisfied and the maximum benefit is guaranteed.

\section{Acknowledgements}

Authorities of Northeastern University of Shenyang and SS Cyril and Methodius of Skopje are acknowledged for long supporting the academic collaboration between Professors Jing and Dimirovski. Also, Dimirovski is grateful to Dogus University for continuing support of his international activities since he joined this university.

\section{REFERENCES}

Baiocchi A, Priscoli F D, et al. (1995). The geometric dynamic channel allocation as a practical strategy in mobile networks with bursty user mobility. IEEE Trans on Veh Technol. 42(1):14-23.

Cheng Jiang, Zhu Shihua, and Dang Anhong.(2001). Channel Assignment Based on Priority Borrowing. Journal of XiAn Jiaotong University. 12(35):1232-1235.

Dang Anhong, Zhu Shihua, Tang Junxiong. (2002). A classified compact dynamic channel assignment for cellular mobile communications systems. Journal of China Institute of Communications. 9(23):43-48.

Guo Jinhuai, Tang Hanping and Li Chao. (2002). A kind of channel assignment in CDMA/TDD mobile communications system. Communication technology. 123:47-49.

Jiang et al .(1994 ). CBWL:a new channel assignment and sharing method for cellular communication systems . IEEE Trans Veh. Technol 43(2):313 - 321 .

Jing Yuanwei and Chen Bing, Georgi M, Khosrow S.(2001). On Leader-Follower Model of Traffic Rate Control for Networks. Control Theory and Applications. 18(6):817-821.

Katzela I, Naghshineh M.(1996 ). Channel assignment schemes for cellular mobile telecommunication systems: a comprehensive survey. IEEE Personal Commun. 3(3):1031.

Li Xu, Liu Feng, Ma Li, Song Junde, Song Mei.(2000). Study and comparison of channel assignment strategy. Telecommunication technology .

Yeung K L, Yum T P.(1994). Compact pattern based dynamic assignment for cellular mobile systems. IEEE Trans on Veh Technol.43(4): 892-896.

Zhang M, Yum T P.(1989). Comparisons of channel assignment strategies in cellular mobile telephone systems.IEEE Trans on Veh Technol. 38(4): 211-215. 